\title{
The $\mathrm{RuO}_{4}$-Catalyzed Ketohydroxylation - Part I. \\ Development, Scope and Limitation.
}

\author{
Bernd Plietker* \\ Organic Chemistry II, Chemistry Faculty, Dortmund University, Otto-Hahn-Str. 6, D-44221 \\ Dortmund, Germany.
}

plietker@pop.uni-dortmund.de

\section{List of Contents}

S-2 General experimental methods

S-3 ${ }^{1} \mathrm{H}$ NMR spectra of the acyloins

\section{S-16 References}




\section{General Remarks.}

Infrared spectra (IR) were recorded as a thin film between $\mathrm{KBr}$-plates. Proton $\left({ }^{1} \mathrm{H}\right.$ NMR, $400 \mathrm{MHz})$ and carbon $\left({ }^{13} \mathrm{C} \mathrm{NMR}, 100.6 \mathrm{MHz}\right)$ nuclear magnetic resonance spectra were recorded in chloroform(d-1) and referenced to the solvent signal. All signal points are listed on a $\delta$-scale in ppm. Gaschromatographic analysis was performed using a DB1-column (carrier gas: helium). Flash-chromatography was done on silica 60 (230-400 mesh) using head pressure by means of compressed air. Petroleum ether refers to that fraction boiling in the range $35-60{ }^{\circ} \mathrm{C}$. Ethyl acetate was purified by distillation over $\mathrm{CaCl}_{2}$ prior to use. $\mathrm{RuCl}_{3}$ was obtained from Aldrich. A stock solution was prepared calculating with $\mathrm{RuCl}_{3}\left(\mathrm{H}_{2} \mathrm{O}\right)_{2}$ and dissolving the catalyst (2.44 g, $10 \mathrm{mmol}$ in $100 \mathrm{~mL}$ water $(0.1 \mathrm{M})$. The deep brown solution can be stored on the bench for weeks without loss of activity. Olefins $17^{\mathrm{i}}, \mathbf{2} \mathbf{1}^{\mathrm{ii}}, \mathbf{2 3 ^ { \mathrm { ii } }}, \mathbf{2 5 ^ { \mathrm { iv } }}, \mathbf{2 7 ^ { \mathrm { v } }}$, $\mathbf{2 9}{ }^{\mathrm{vi}}$ and $33^{\mathrm{vii}}$ were prepared according to literature procedures. All other starting material were purchased from commercial suppliers and used without further purification. In cases where the regioselectivity of the oxidation was better than $90: 10$ only the spectral data of the major isomer are reported. Regioselectivities were determined by GC- or NMR-integration of the crude product mixture. 
1H NMR spectra
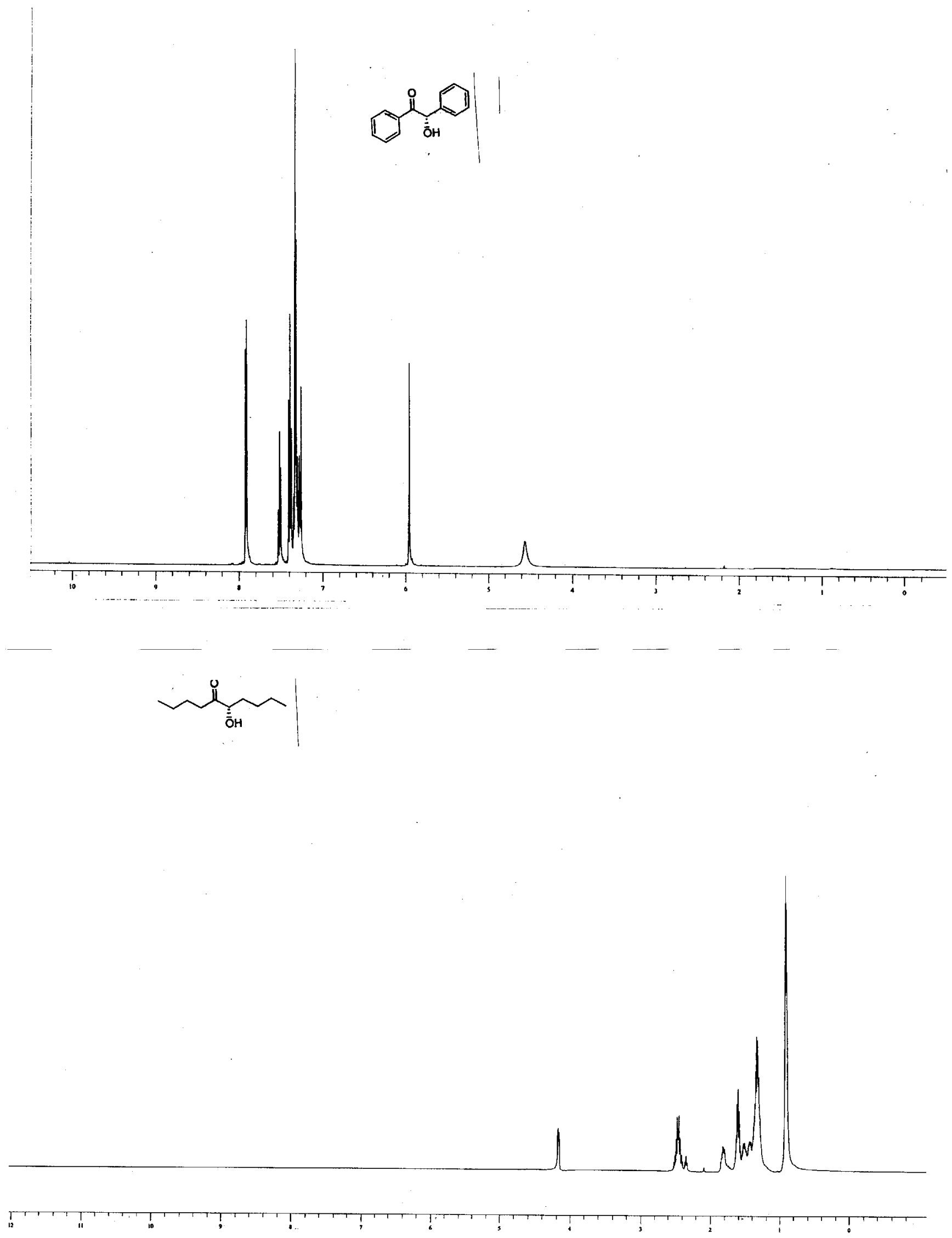

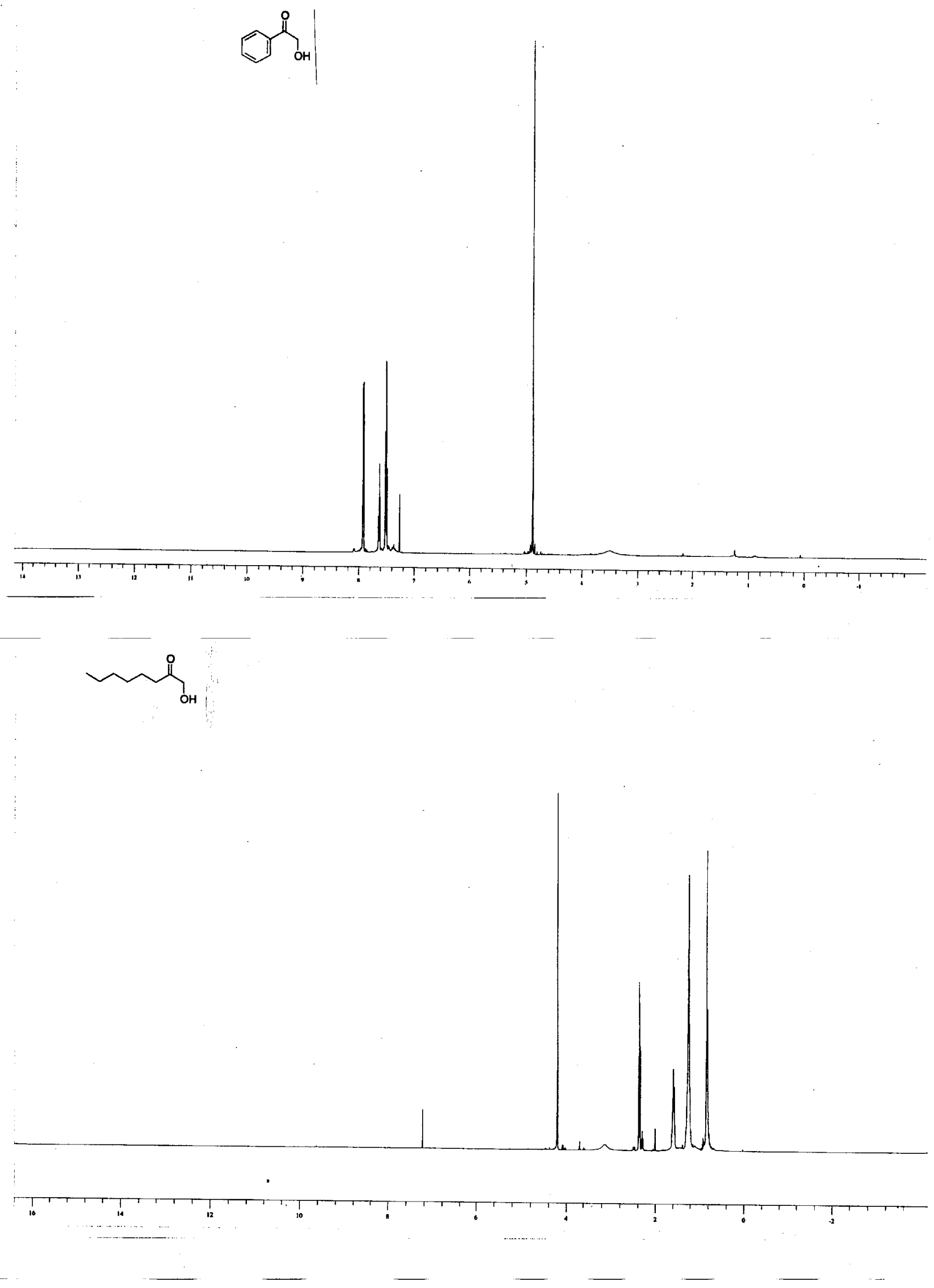

S-4 

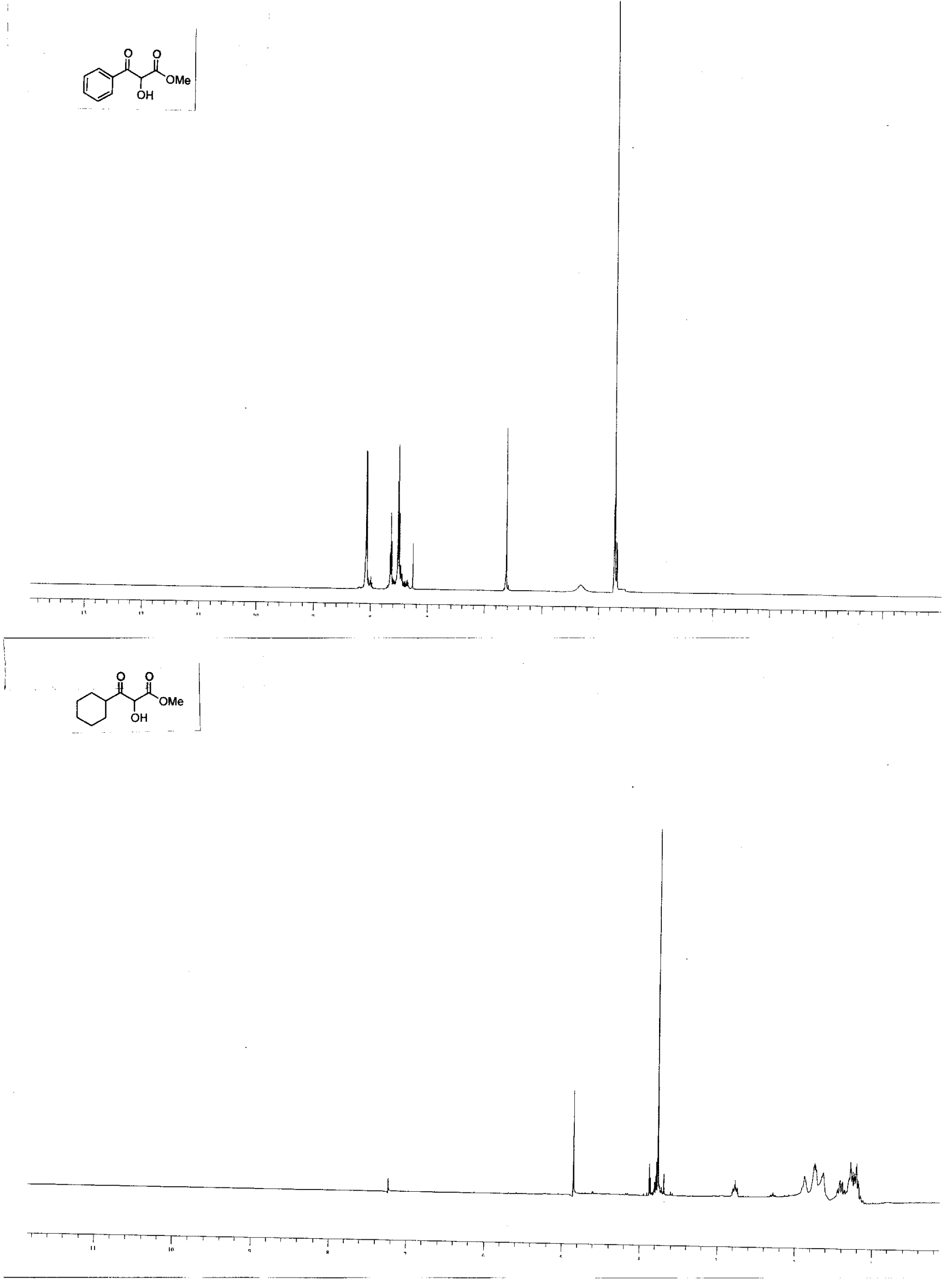

S-5 
onst

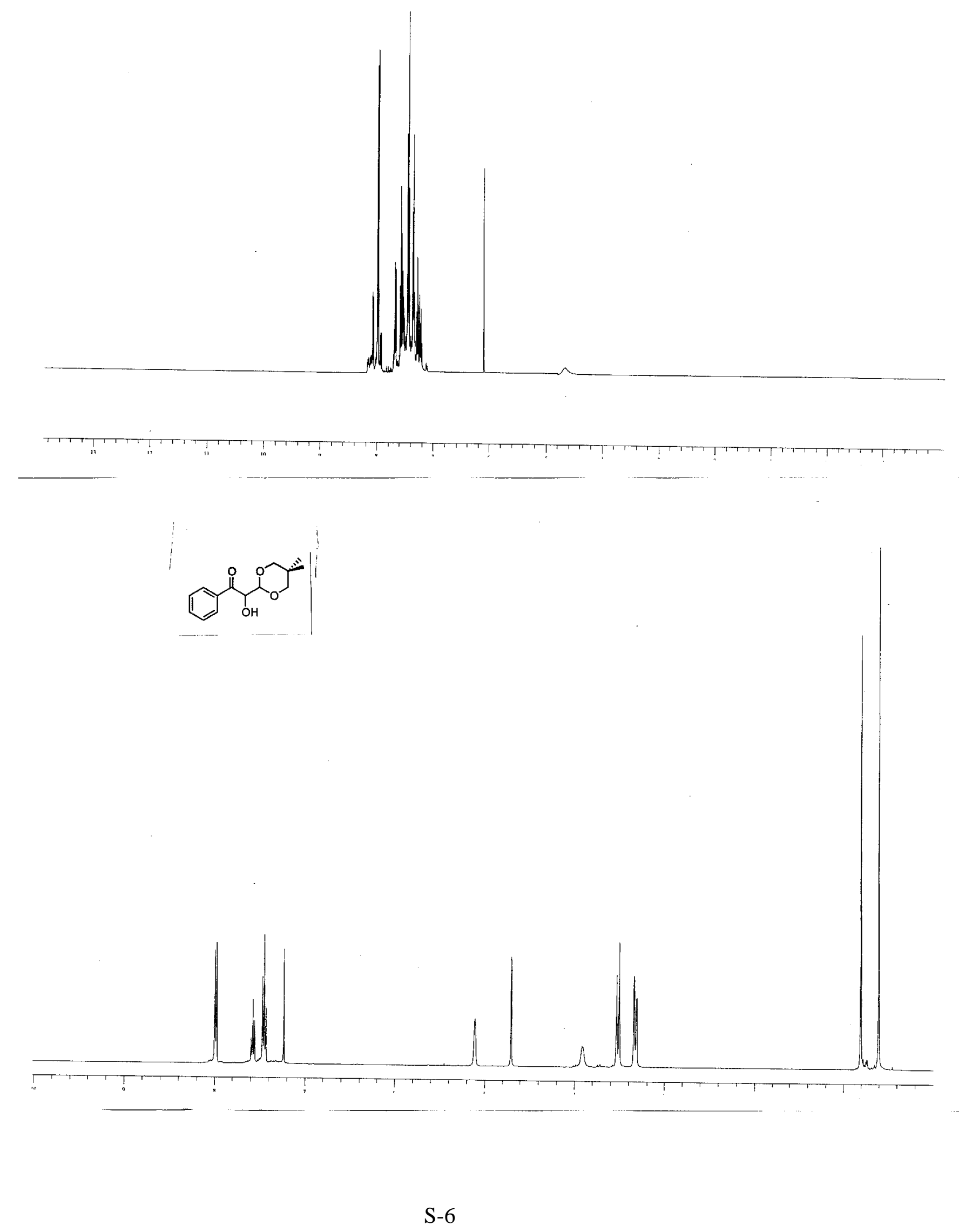




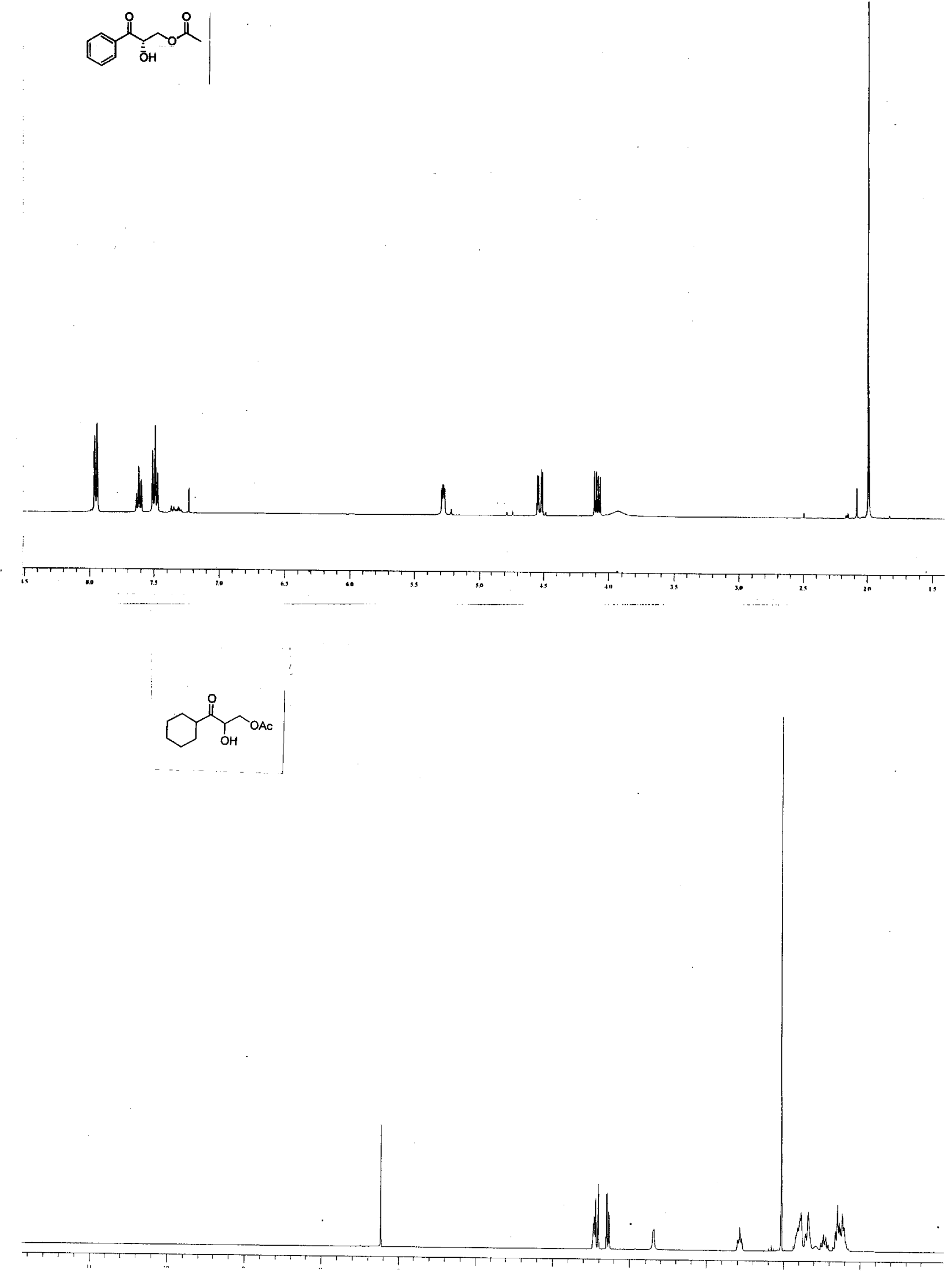

S-7 


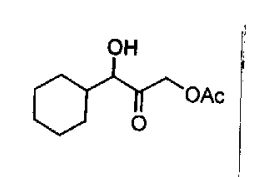
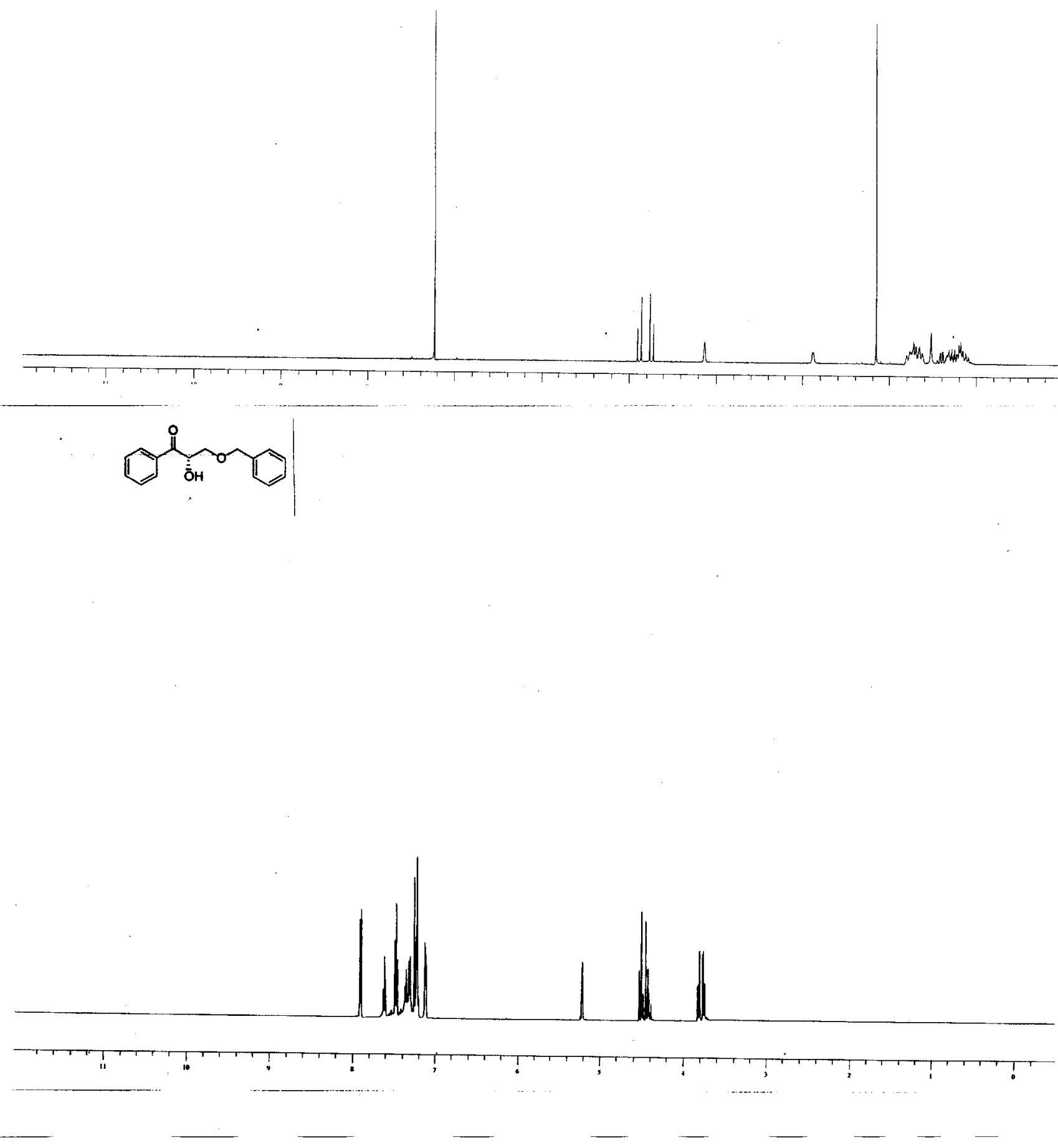

S-8 
ㅇำํำ 0
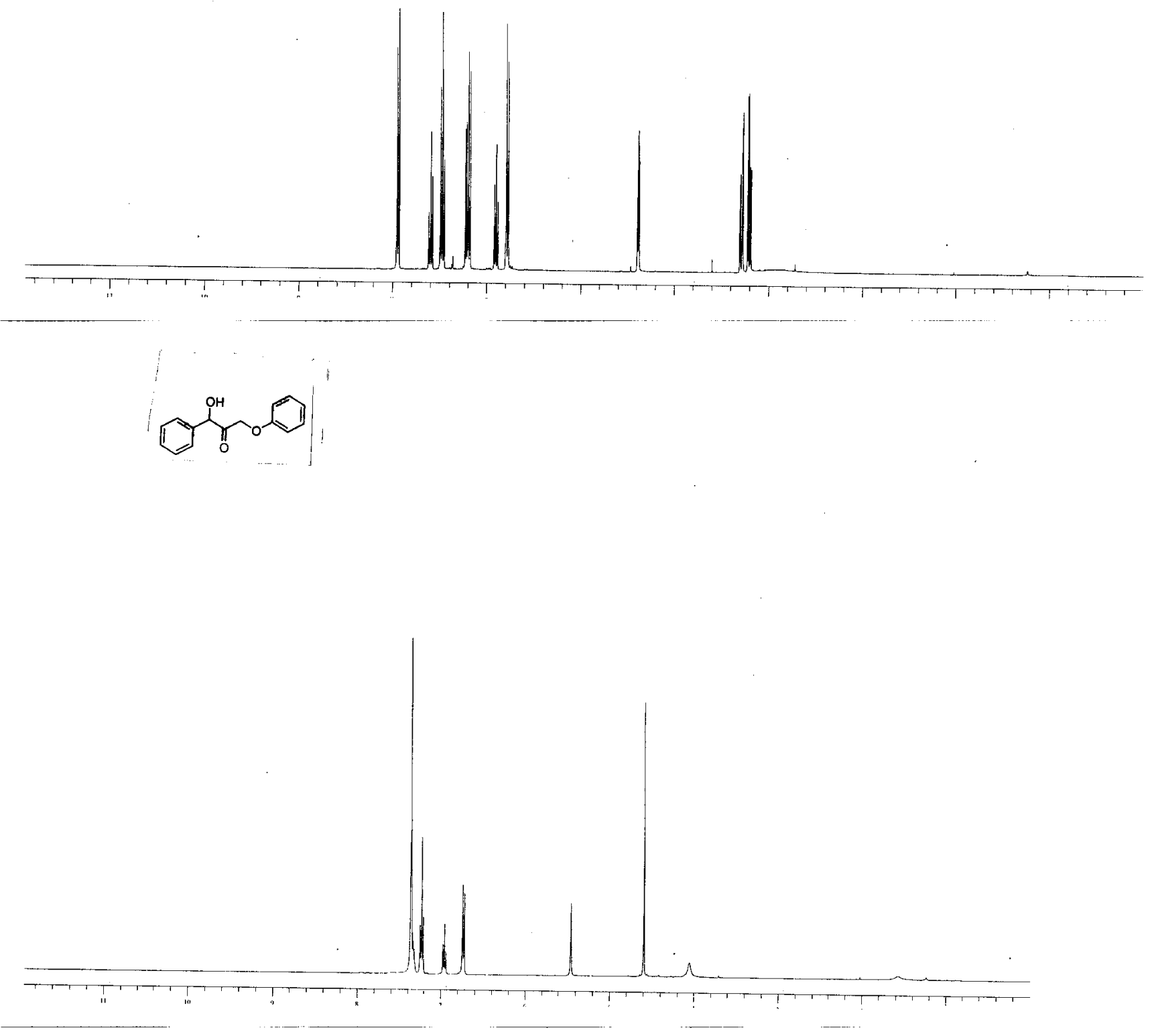

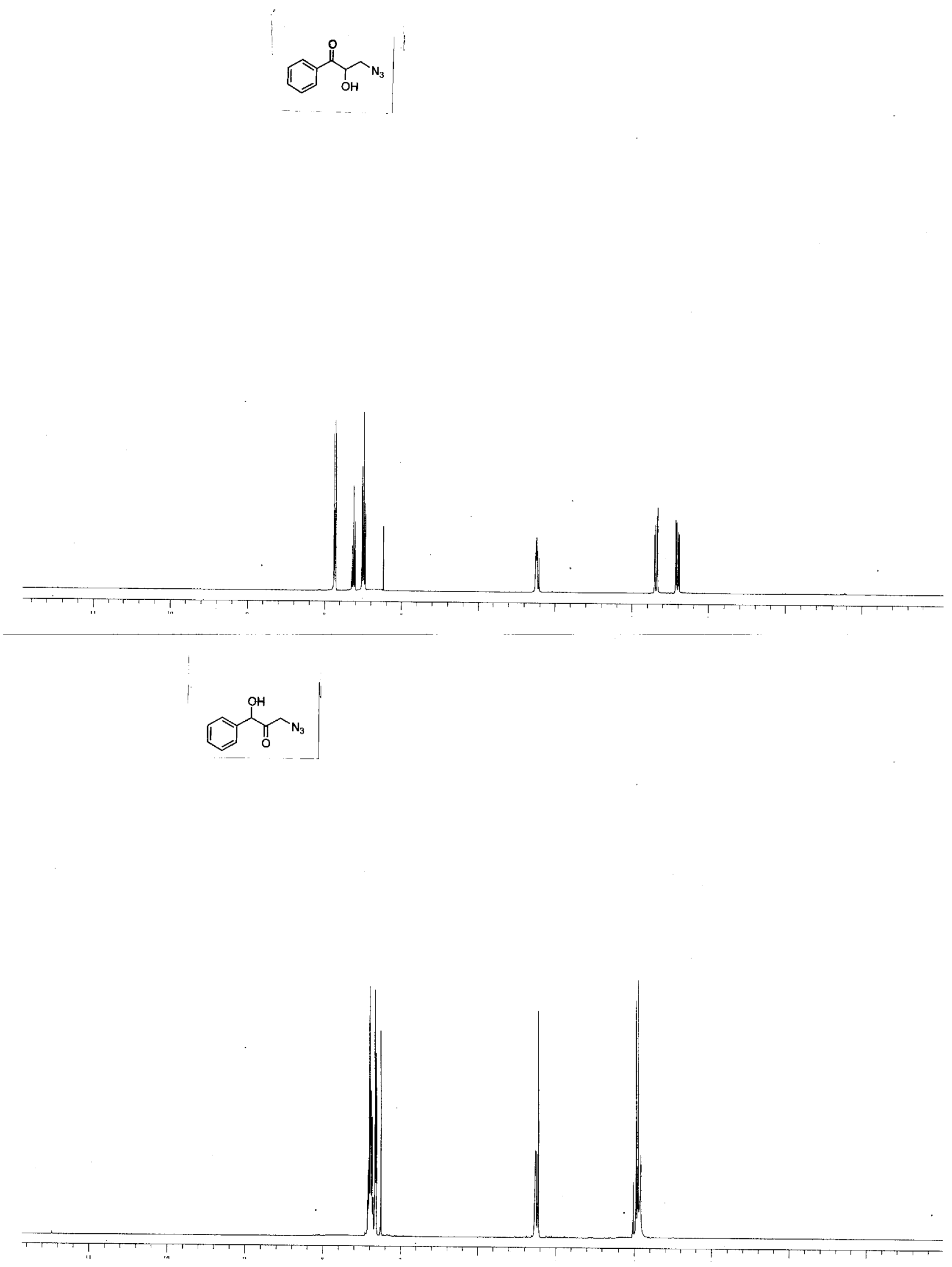

S-10 

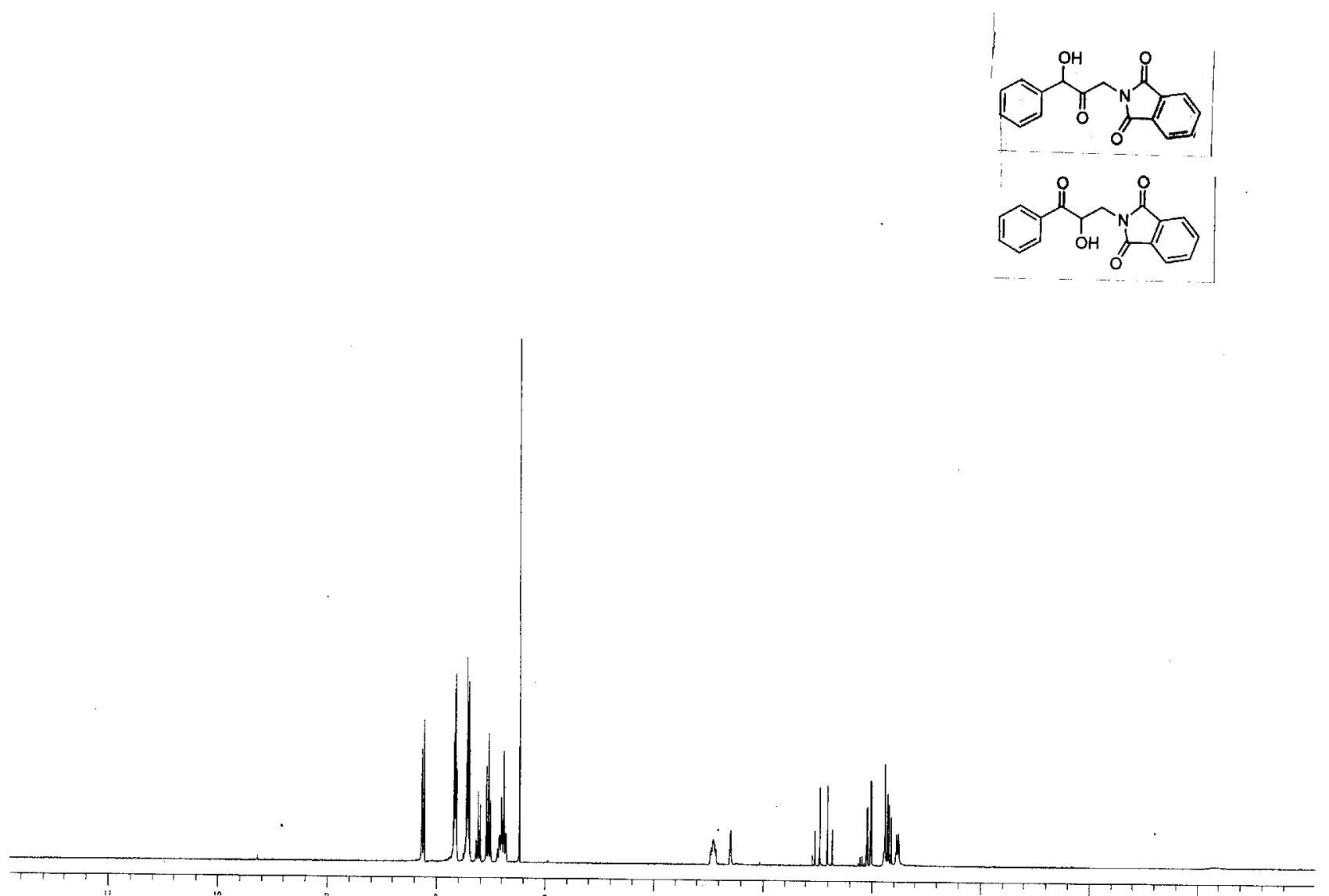

$$
\text { |c }
$$

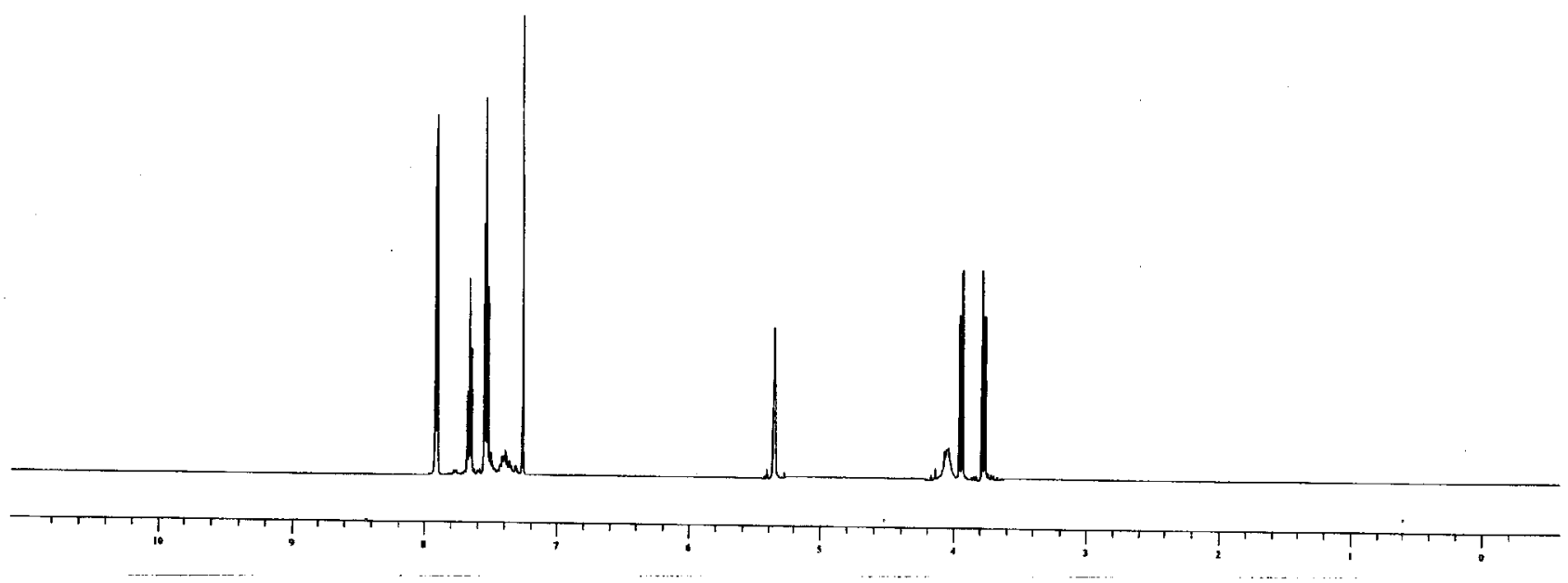

S-11 


$$
\text { (1) }
$$
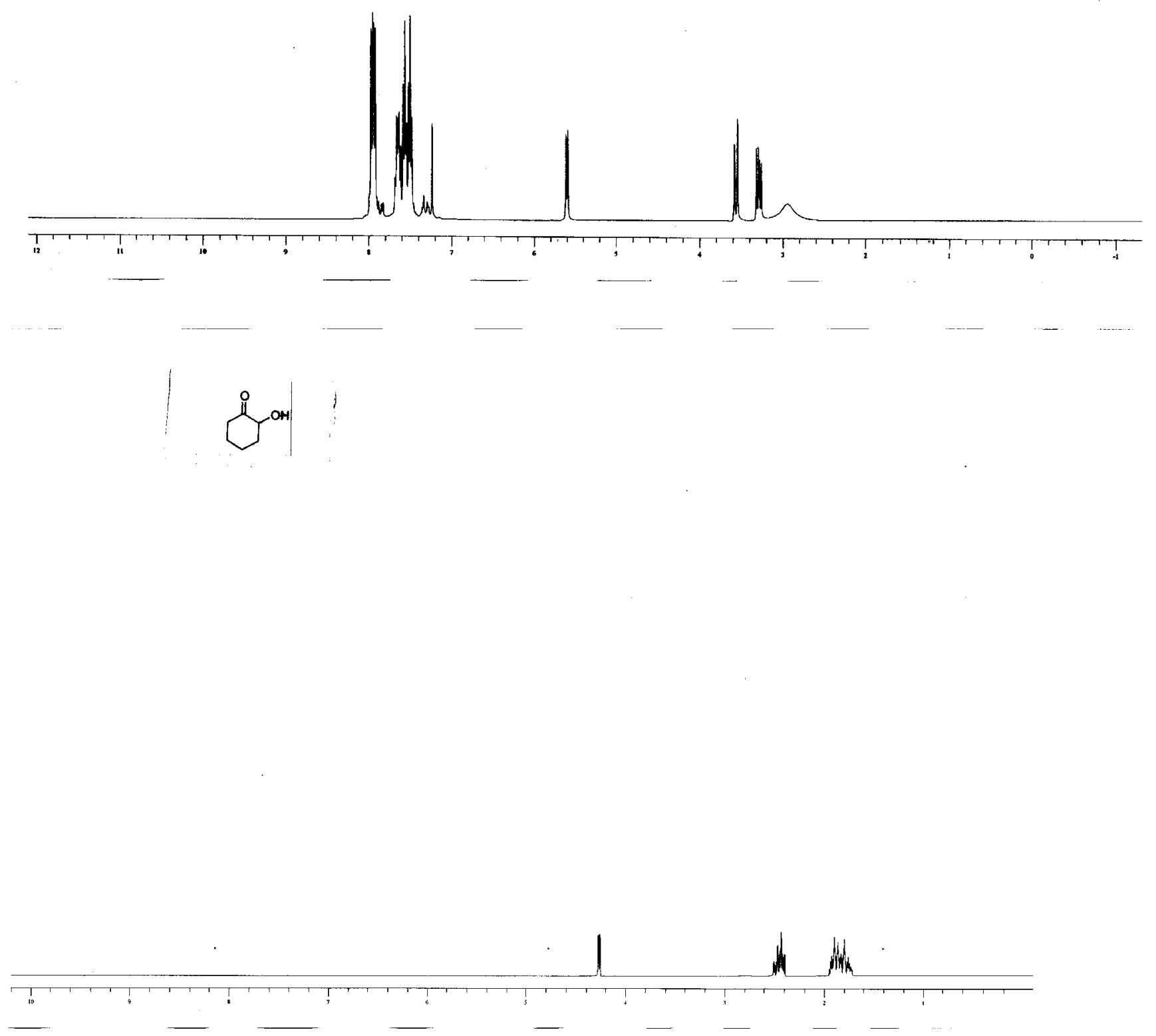
80
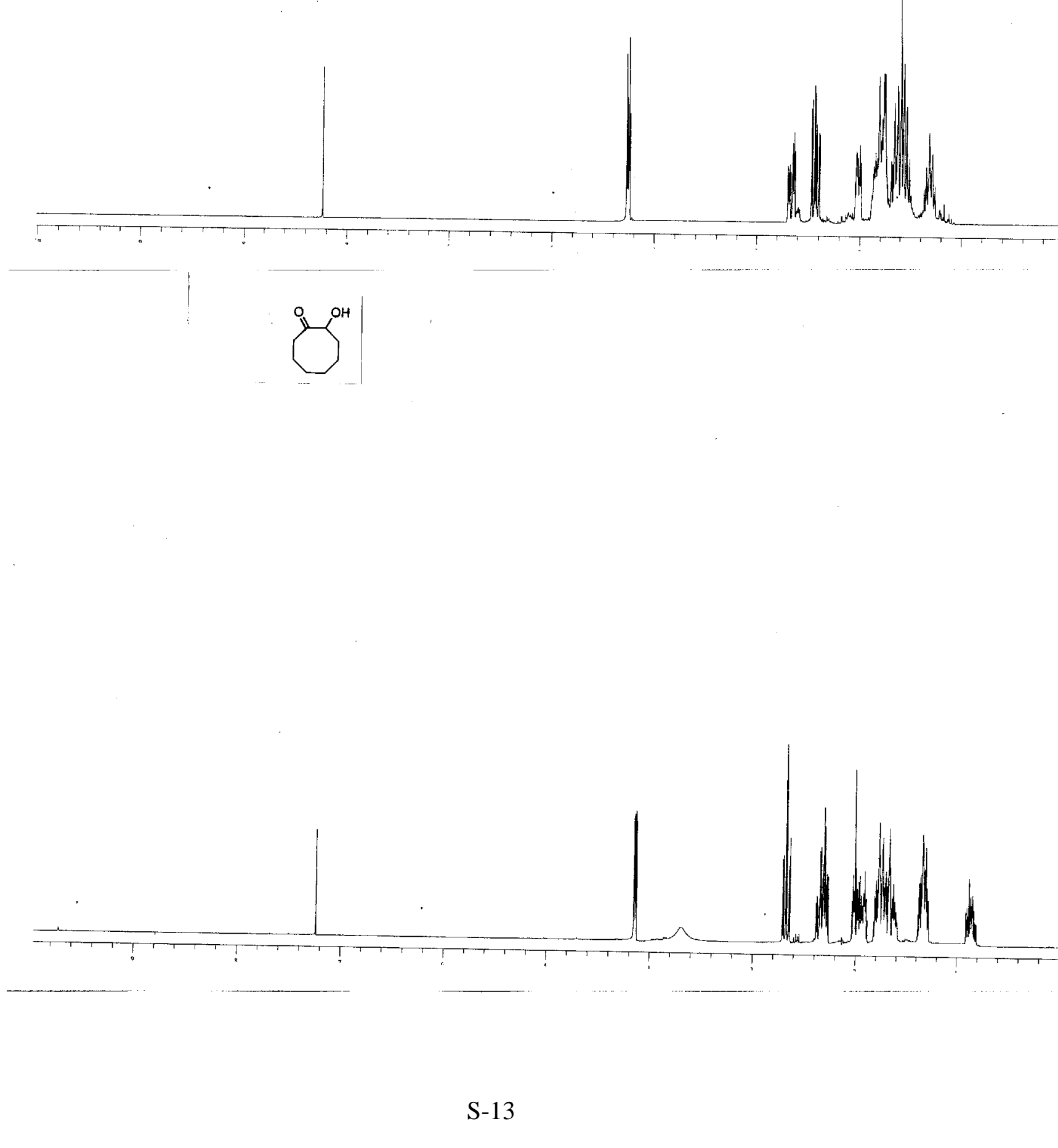


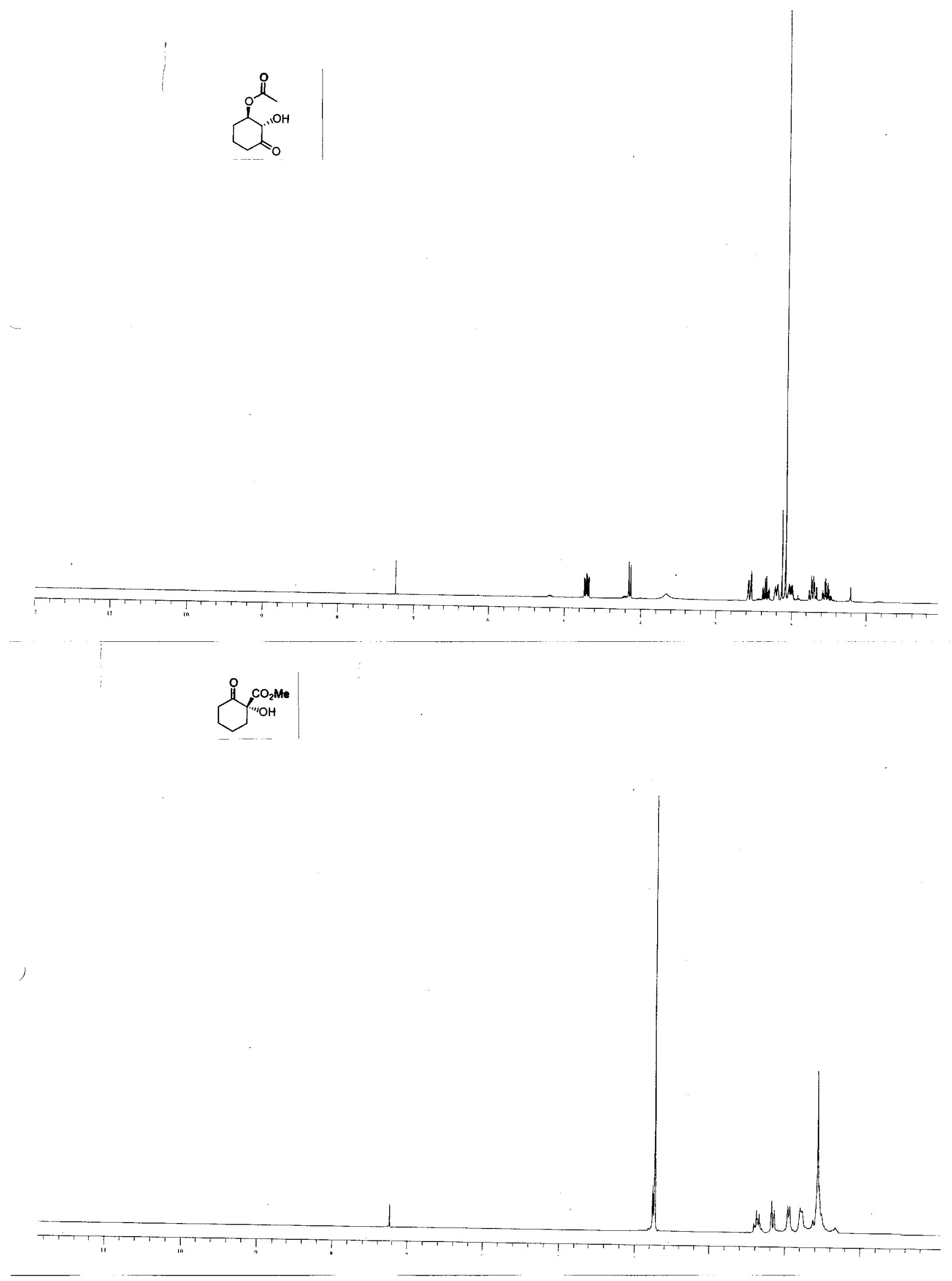


(y)

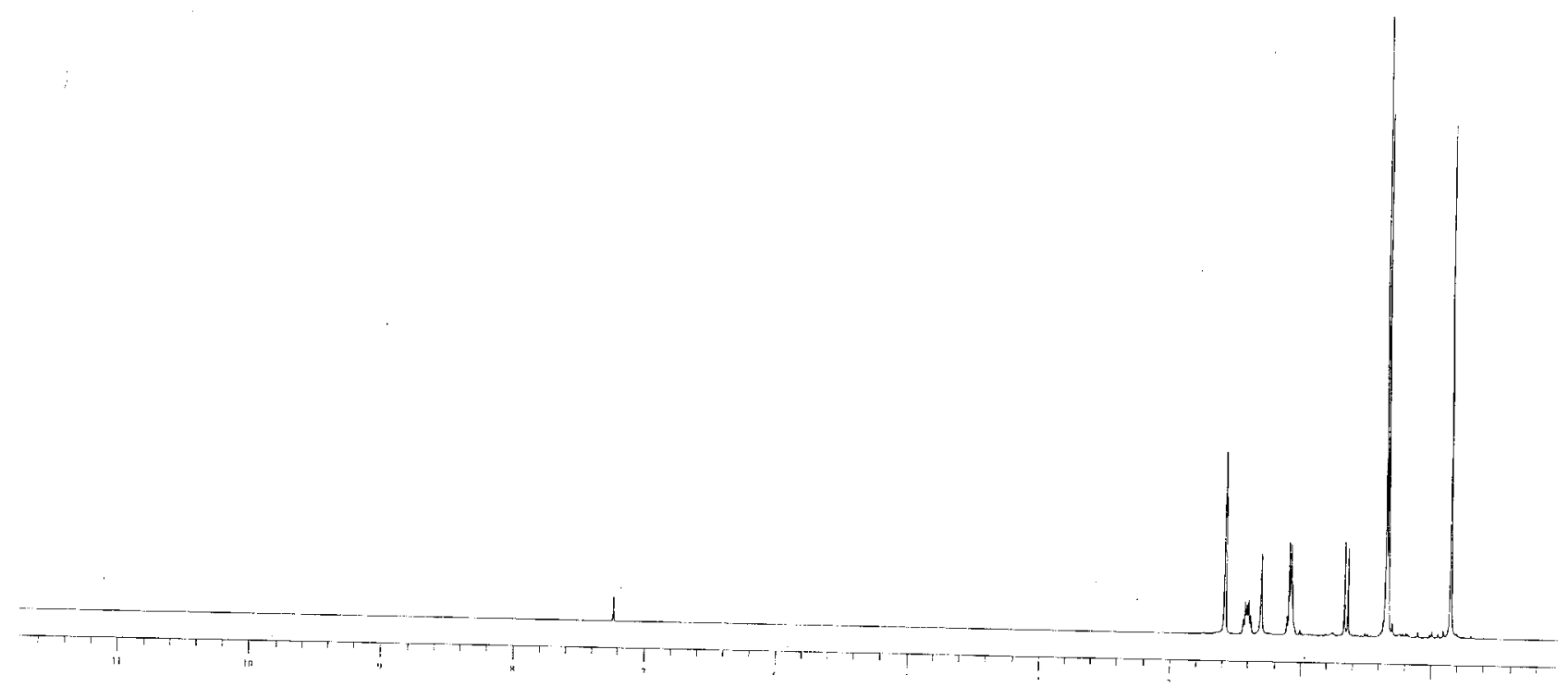




\section{References}

(i) Sarkar, A.; Yemul, O. S.; Bandgar, B. P.; Gaikwad, N. B.; Prakash, P. P.; Org. Prep. Proced. Int. 1996, 28, 613.

(ii) Persson, E. S. M.; Bäckvall, J.-E.; Acta Chem. Scand. 1995, 49, 899.

(iii) Denmark, S. E.; Edwards, J. P.; J. Org. Chem. 1991, 56, 6974.

(iv) Hurd, C. D.; Schmerling, L.; J. Am. Chem. Soc. 1937, 59, 107.

(v) Manhart, E.; von Werner, K.; Synthesis 1978, 705.

(vi) Gensler, W. J.; Rockett, J. C.; J. Am. Chem. Soc. 1955, 77, 3262.

(vii) Pyne, S. G.; David, D. M.; Dong, Z.; Tetrahedron Lett. 1998, 39, 8499. 